\title{
IL-4 Suppresses UVB-induced Apoptosis in Skin
}

\author{
Hayoung Hwang ${ }^{1,3}$, Soo-Young Choi ${ }^{2}$ and Tae Yoon Kim ${ }^{1, *}$ \\ ${ }^{1}$ Laboratory of Dermatology-Immunology, Catholic Research Institute of Medical Science, College of Medicine, \\ The Catholic University of Korea, Seoul 137-701, Korea \\ ${ }^{2}$ Department of Biomedical Sciences, Hallym University, Chunchon 200-702 Korea \\ ${ }^{3}$ Dermatology Branch, National Cancer Institute, National Institute of Health, Bethesda, Maryland, USA
}

Received 4 August 2006, Accepted 24 August 2006

In this study, cutaneous role of IL-4 in UVB-induced apoptosis was investigated using transgenic mice with skinspecific expression of IL-4 (IL-4 Tg mice). The transgenic mice did not show any gross clinical abnormalities. However, epidermis was thickened and increased MHC class II positive cells were detected as well as enhanced expression of inflammatory cytokines such as IL-1 and TNF- $\alpha$ in skin. In addition, histological analysis revealed increased infiltration of lymphocytes, acanthosis, hyperkeratosis, and parakeratosis in skin of IL-4 Tg mice. The physiological effect of IL-4 overexpression in skin against environmental stimulus such as UVB was investigated by irradiating wild-type and IL-4 Tg mice with UVB followed by evaluation of apoptosis. The result demonstrated suppressed apoptosis in epidermis of IL-4 Tg mice compared with wild-type mice. To further assess anti-apoptotic function of IL-4 in keratinocytes, stable cell clones were made where IL-4 was constitutively overexpressed and examined for UVB-induced apoptosis. The results showed that apoptosis was remarkably decreased in IL-4 over-expressing cell clones compared with that in mock transfected cells. Collectively, data presented here shows that IL-4 has an inhibitory effect against UVB-induced apoptosis in keratinocytes, suggesting that IL-4 may be an important regulator in cutaneous immunity against UVB.

Keywords: Apoptosis, IL-4, Keratinocyte, Transgenic mice, UVB

* To whom correspondence should be addressed.

Tel: 82-2-590-2625; Fax: 82-2-3482-8261

E-mail: tykimder@catholic.ac.kr

\section{Introduction}

Diverse stimuli such as viral infection, bacterial invasion, toxins, and radiation exist in environment, and induce stressresponsive immune reaction in human. Among them, ultraviolet radiation (UV) represents one of the most important environmental factor affecting humans. In particular, UVB with a wavelength range between 290 and $320 \mathrm{~nm}$ has been regarded as major inducing factor of the generation of skin cancer, suppression of the immune response as well as premature skin aging. Besides, exposure of UVB induces the formation of sunburn cells within the epidermis, which undergo programmed cell death and show erythema and edema as a result of increased vascular permeability (Ullrich et al., 2000; Cleaver et al., 2002; Kulms et al., 2002; Schwarz et al., 2002c).

IL-4 produced by activated T cells, basophils, and mast cells plays diverse roles in the regulation of the immune systems. IL-4 promotes T cells differentiation toward the Th2 cell type, improves the antigen presenting capacity of B cells by inducing the expression of MHC class II gene, and increases survival of B cells (Mori et al., 2000; Boothby et al., 2001; Liu et al., 2004). Furthermore, IL-4 is involved in the proliferation and maturation of mast cells and macrophages (Lorentz et al., 2001). In addition, IL-4 has been shown to act as a survival factor by preventing apoptosis in a variety of cell types, including T cells, B cells, and mast cells (Morris et al., 2002; Lorentz et al., 2005). In skin, IL-4 has been considered as a factor that is associated with the pathogenesis of atopic dermatitis and it was previously reported that transgenic mice expressing IL-4 in skin exhibited a clinical symptom similar to atopic dermatitis (Chan et al., 2001; Elbe-Burger et al., 2002).

In this study, we investigated the role of IL-4 in skin under UVB irradiation using transgenic mice with its skin-specific expression. Our data presented here demonstrate that IL-4 plays an anti-apoptotic function in skin upon UVB irradiation and this provides further understanding of the cutaneous role of IL-4. 


\section{Materials and Methods}

Generation of IL-4 transgenic mice. IL-4 transgene construct contains upstream elements of human keratin-14 (K14) gene, rabbit globin intron, cDNA encoding mouse IL-4, and K14 polyadenylation sequence (Fig. 1). The linearized transgene construct by KpnI/SalI digestion was used for microinjection into oocytes of BDF mice, which were implanted in pseudopregnant mice. The offspring were examined for the incorporation of IL-4 by PCR-genotyping using genomic DNA isolated from tail clippings. Mice at 8-12 weeks of age were used in experiments.

Genotyping. Transgenic mice were screened for genomic incorporation of the transgene by PCR. Briefly, $1-2 \mathrm{~mm}$ of tail was incubated in $0.3 \mathrm{ml}$ lysis buffer (100 mM Tris-HCl, $\mathrm{pH} 8.5,5 \mathrm{mM}$ EDTA, $\mathrm{pH}$ $8.0,0.2 \% \mathrm{SDS}, 200 \mathrm{mM} \mathrm{NaCl})$ containing $250 \mu \mathrm{g} / \mathrm{ml}$ of proteinase $\mathrm{K}$ (Sigma) at $55^{\circ} \mathrm{C}$ for overnight. After incubation, one volume of isopropanol was added and genomic DNA was precipitated by centrifugation, washed in $70 \%$ ethanol, and resuspended in TE, followed by PCR using primers corresponding to the sequences of flanking region of $\mathrm{K} 14$ and IL-4 under the following conditions: $3 \mathrm{~min}$ at $94^{\circ} \mathrm{C}$; 35 cycles of $94^{\circ} \mathrm{C}$ for $30 \mathrm{~s}, 60^{\circ} \mathrm{C}$ for $30 \mathrm{~s}, 72^{\circ} \mathrm{C}$ for $1 \mathrm{~min}$; and a $7 \mathrm{~min}$ at $72^{\circ} \mathrm{C}$. The resulting PCR product was analyzed in $1 \%$ agarose gel. The sequence of the primers used for genotyping are as follows:

\section{hK14TR: ttccagtgggatctgtgtcc \\ mIL-4: tactacgagtaatccatttgcatg}

RT-PCR. Total RNA extracted using Trizol reagent (Invitrogen) was used for reverse transcription, which was subjected to PCR

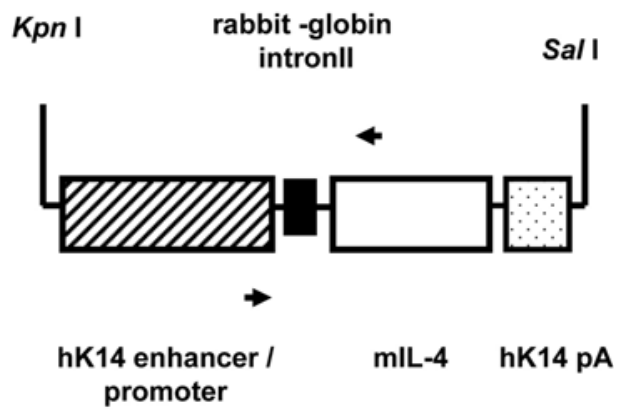

Fig. 1. IL-4 transgene construct. The transgene construct was linearized by KpnI/SalI digestion, as described in Materials and Methods, and used for microinjection into oocytes. Arrows indicate the primers used for genotyping.

using primers specific to IL- $1 \alpha$, IL- $1 \beta$, IL- 4 , and TNF- $\alpha$ in $28-32$ cycles. Glyceraldehydes-3-phosphate dehydrogenase (GAPDH) was employed as an internal control. The amplification profile was $30 \mathrm{~s}$ at $94^{\circ} \mathrm{C}, 30 \mathrm{~s}$ at $60^{\circ} \mathrm{C}$, and $30 \mathrm{~s}$ at $72^{\circ} \mathrm{C}$.

Northern blot analysis. For northern blot analysis, twenty microgram of total RNA or PCR-amplified cDNA was electrophoresed and transferred onto nylon membrane, followed by hybridization in hybridization buffer (6X SSPE, 1\% SDS, 10X Denhardts solution, $20 \mu \mathrm{g} / \mathrm{ml}$ yeast tRNA and $50 \mu \mathrm{g} / \mathrm{ml}$ salmon sperm DNA), washing and exposure onto the X-ray film.

Measurement of ear thickness. Ear thickness of transgenic mice and non-transgenic mice was measured with mitutoyo's gauge.
(A)

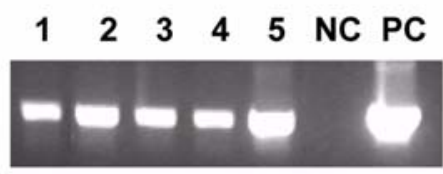

(C)

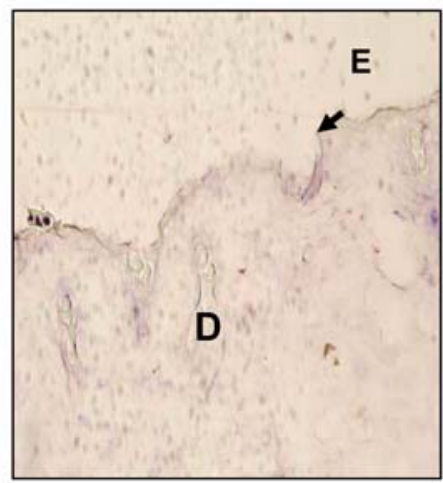

Wild type
(B)

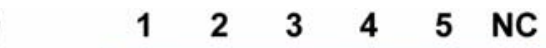

IL-4

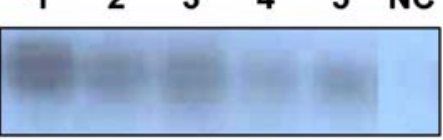

GAPDH

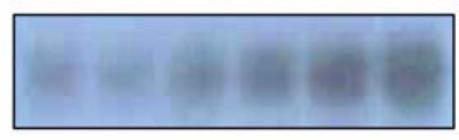

(D)

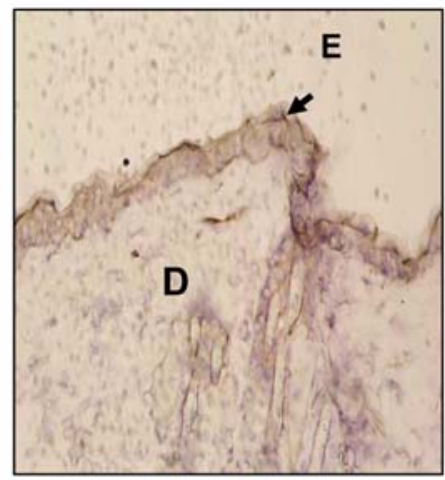

IL-4 Tg

Fig. 2. Screening of IL-4 transgenic mice. (A) Detection of IL-4 transgene by genotyping analysis. NC means negative control and PC, positive control. Lanes 1-5 represent individual offsprings. (B) Detection of IL-4 transcript in skin. Lanes 1-5 represent individual offsprings (C) Detection of IL-4 protein in skin. (D) D and E means dermis and epidermis, respectively. 
Immunohistochemistry and $\mathbf{H \& E}$ staining. Biopsies obtained from skin of IL-4 transgenic mice and non-transgenic mice were fixed in neutralized buffered formalin (NBF) and embedded in paraffin. Five micrometer tissue sections were obtained and used for hematoxylin-eosin (H\&E) staining and also for detection of IL4 expression using $\mathrm{ABC}$ reagent (DAKO) according to manufacturer's instructions. The epidermal hyperplasia was assessed by measuring vertical thickness of epidermis. The thickness was measured in six randomly selected regions using an image analysis system with a microscope (Olympus). The thickness of epidermis was defined as the length from the basal layer to the stratum corneum.

Flow cytometry analysis. Mouse epidermal cells $\left(1 \times 10^{6}\right)$ were prepared from ear and incubated with primary antibody to MHC class II (Pharmingen) for $1 \mathrm{~h} 30 \mathrm{~min}$ with gentle shaking in ice. After washing in PBS, cells were incubated with FITC-conjugated secondary antibody (Serotec) for $1 \mathrm{~h}$ and subsequently used for FACS analysis.

Cell culture and ELISA. Mouse keratinocyte cell line, Pam212, was grown in DMEM media (Invitrogen) supplemented with 10\% FBS and $1 \%$ of penicillin and streptomycin. For the preparation of stable cell line, Pam 212 cells were transfected with pLNCX-IL-4 or pLNCX plasmid. Forty-eight hours after transfection, Geneticin (Invitrogen) was added at $0.5 \mathrm{mg} / \mathrm{ml}$ to the culture medium and Geneticin-resistant clones were selected. For measurement of IL-4 concentration, $1 \times 10^{4}$ cells were seeded in 96 well plate and, twenty-four hours later, culture supernatant was harvested followed by determination of IL-4 concentration using ELISA kit (R\&D).

UVB irradiation. Mice at $8-12$ weeks of age were shaved to expose back skin and, $24 \mathrm{hs}$ later, were irradiated with UVB at $2 \mathrm{~kJ} /$ $\mathrm{m}^{2}$. Twenty-four hrs after irradiation, mice were sacrificed and the irradiated skin was used for TUNEL assay. For UV irradiation on cells, Pam 212 cells were seeded at $2 \times 10^{5}$ in $60 \mathrm{~mm}$ dish plate, and 24 hrs later, washed with PBS, and then irradiated with UVB at 200 $\mathrm{mJ} / \mathrm{cm}^{2}$. The cells harvested $24 \mathrm{hrs}$ after post-irradiation were used for FACS analysis or TUNEL assay.

Detection of apoptosis. TUNEL assay was carried out using in situ cell death detection kit, POD (Roche) according to manufacturer's instructions. The number of TUNEL positive cell was counted in equal area of six randomly selected regions. For FACS analysis, $1 \times 10^{6}$ cells were incubated with annexin V-Fluous (Roche) for $1 \mathrm{~h}$ and annexin V-positive cells were detected using flow cytometry.

\section{Results}

Generation of transgenic mice expressing IL-4 in skin. To investigate the role of IL-4 in skin, IL-4 transgenic mice (IL-4 $\mathrm{Tg}$ ) were generated using human keratin-14 (K14) promoter for keratinocyte-specific expression (Fig 1). The IL-4 gene incorporation successfully occurred in one founder mouse and the founder was mated with non-transgenic mice to produce offspring that were screened by RT-PCR (Fig 2A and B). Furthermore, selective expression of IL-4 in epidermis of

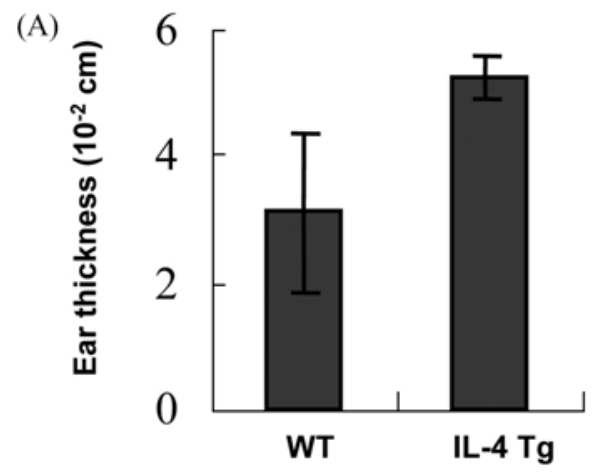

(B)

Exp Group $\quad \%$ of I-A ${ }^{d}$ (MK-D6)

\begin{tabular}{cc}
\hline WT & $1.5 \%$ \\
IL-4 Tg & $4 \%$
\end{tabular}

(C)

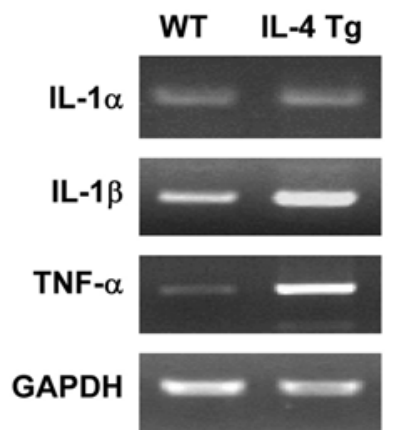

Fig. 3. Characterization of IL-4 transgenic mice. (A) The increased of ear thickness in IL-4 Tg mice. The value represents $\times 10^{-2} \mathrm{~cm}$. (B) Increase of MHC-class II positive cells in epidermis of IL-4 transgenic mice. Epidermal cells isolated from ear were incubated with FITC conjugated I-A ${ }^{\mathrm{d}}$ antibody for $1 \mathrm{~h}$, as described in Materials and Methods, followed by flow cytometry. (C) The increased expression of IL-1 $\beta$ and TNF- $\alpha$ in skin of IL-4 Tg mice.

transgenic mice was demonstrated by immunohistochemistry. As shown in Fig. 2C, the expression of IL-4 was detected in epidermis of transgenic mice whereas non-transgenic mice did not show any positive staining for IL-4.

Effects of IL-4 expression in epidermis. IL-4 $\mathrm{Tg}$ mice did not show any apparent gross clinical abnormalities upon observation from birth except the average thickness of skin was slightly increased (Fig. 3A).

In order to further examine the effect of IL-4 in epidermal cells of transgenic mice, we analyzed the expression of MHC class II in epidermal cells as well as inflammatory cytokines such as IL-1 and TNF- $\alpha$ in skin of transgenic mice. FACS analysis of epidermis separated from IL-4 Tg mice demonstrated approximately 2-3 fold higher expression of MHC class II in the epidermal cells compared to that from wild-type mice (Fig. 3B). In addition, increased transcription of TNF- $\alpha$ and 

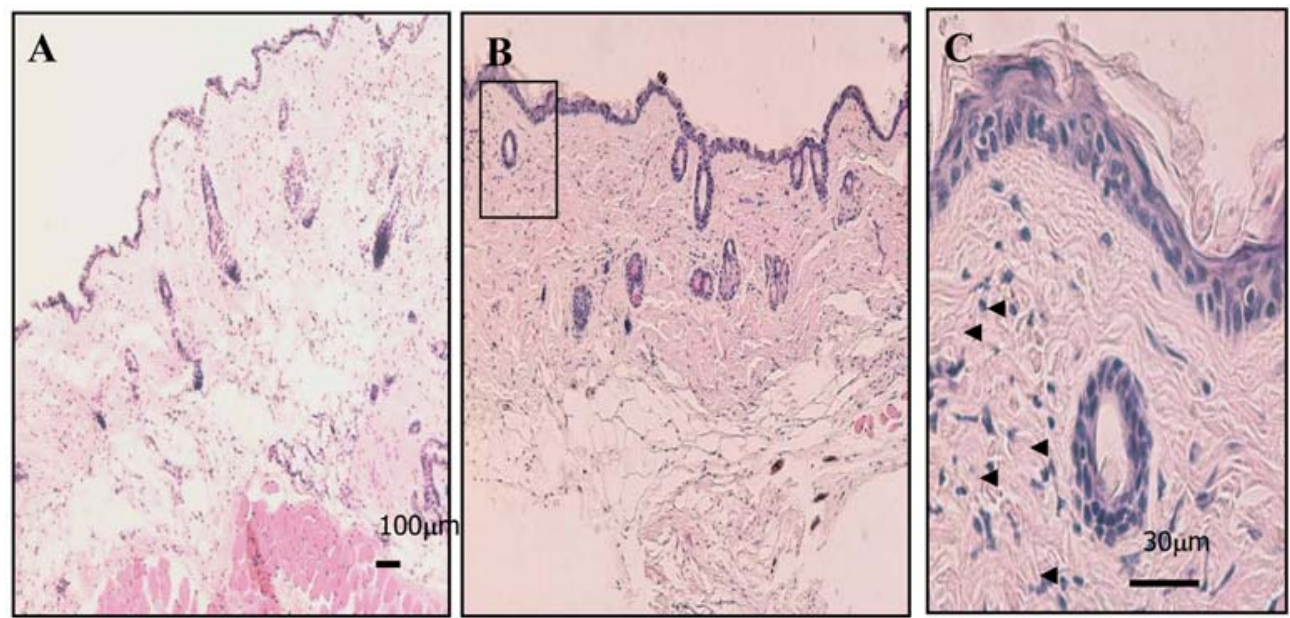

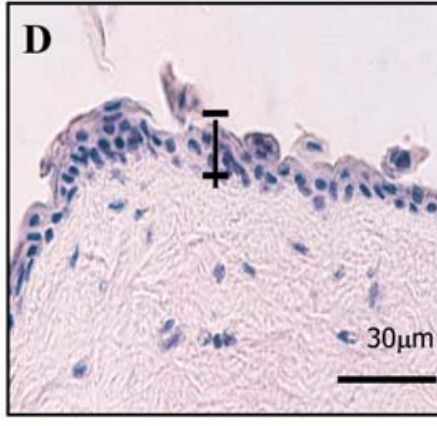

WT

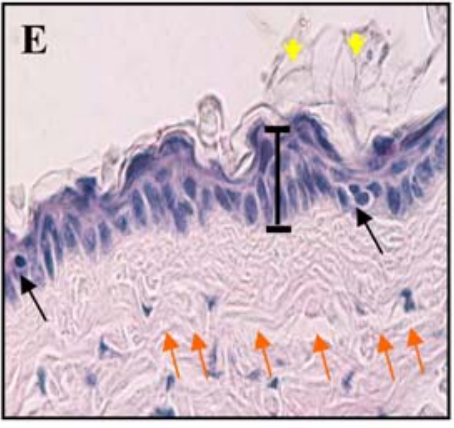

IL-4 Tg

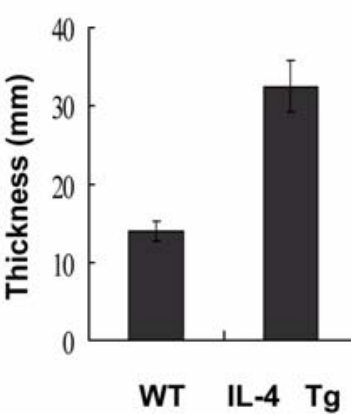

Fig. 4. Histochemical analysis of skin. Five micrometer sections of skin biopsies from IL-4 Tg and wild-type mice were used for H\&E staining. (A) and (B); $\times 100,(C),(D)$, and (E); $\times 200$. The graph represents the average epidermal thickness of six randomly selected regions. The thickness of epidermis was defined as the length from the basal layer to the stratum corneum.

IL-1 $\beta$ was detected in skin from IL-4 Tg mice (Fig. 3C).

Histological examination of skin from IL-4 Tg mice revealed infiltration of immune cells in skin (black arrow in Fig. $4 \mathrm{C}$; Fig. 4C is magnified picture of a square in Fig. 4B.) as well as thickened epidermis (Fig. 4D and E; the graph to the right of Fig. 4E represents the thickness of epidermis in value). Besides, similar to previous observation by Elbe-Burger et al. (2002), acanthosis (black arrow), hyperkeratosis (orange arrow), and parakeratosis (yellow arrow) were detected in skin of IL-4 Tg mice (Fig. 4E).

\section{Reduction of apoptotic cells after UVB irradiation in IL-4} Tg mice. Previous reports by others (Keegan et al., 1998; Stack et al., 1998; Morris et al., 2000) have suggested anti-apoptotic function of IL-4. Since skin is the part of the body which is constantly exposed to UV irradiation, we investigated the role of IL-4 in UV-mediated apoptosis. Experiments were performed where both IL-4 $\mathrm{Tg}$ and wild-type mice were irradiated with $2 \mathrm{~kJ} / \mathrm{m}^{2}$ of UVB, sacrificed $24 \mathrm{~h}$ after irradiation, and examined for apoptosis by TUNEL assay as shown in Fig. 5 A-D. The data revealed less number of apoptotic cells in skin of IL-4 Tg mice compared with that of wild-type mice. Although the reduction of apoptotic cells was not dramatic, the difference was statistically significant, showing $20 \%$ reduction of relative TUNEL-positive cells in IL-4 Tg mice compared to wild-type upon UVB irradiation in Fig. 5E. Meanwhile, UVB irradiation did not cause further thickening of epidermis in IL-4 Tg mice (Fig. 5F). The data presented here suggests an inhibitory role of IL-4 in UVB-induced apoptosis in skin.

Anti-apoptotic function of IL-4 in mouse keratinocytes upon UVB irradiation. We have shown above that IL-4 might play a role in inhibiting UVB-induced apoptosis in skin. Sine the inhibitory effect was not dramatic, albeit statistically significant, we further investigated the anti-apoptotic function of IL-4 in vitro using mouse keratinocytes where much higher expression of IL-4 was present. A mouse keratinocyte cell line, Pam212, was transfected with IL-4-expressing vector and stable cell clones expressing IL-4 were selected and characterized as shown in Fig. 6. Three cell clones (Pam212/IL-4) were characterized and used for further analysis. In order to investigate the effect of UVB irradiation on IL-4-expresssing keratinocyte clones, Pam212/IL-4 cells were irradiated with UVB $\left(200 \mathrm{~mJ} / \mathrm{cm}^{2}\right)$ followed by detection of apoptotic cells by TUNEL and FACS analysis. The representative figures from experiments with three cell clones were shown in Fig. 7. 

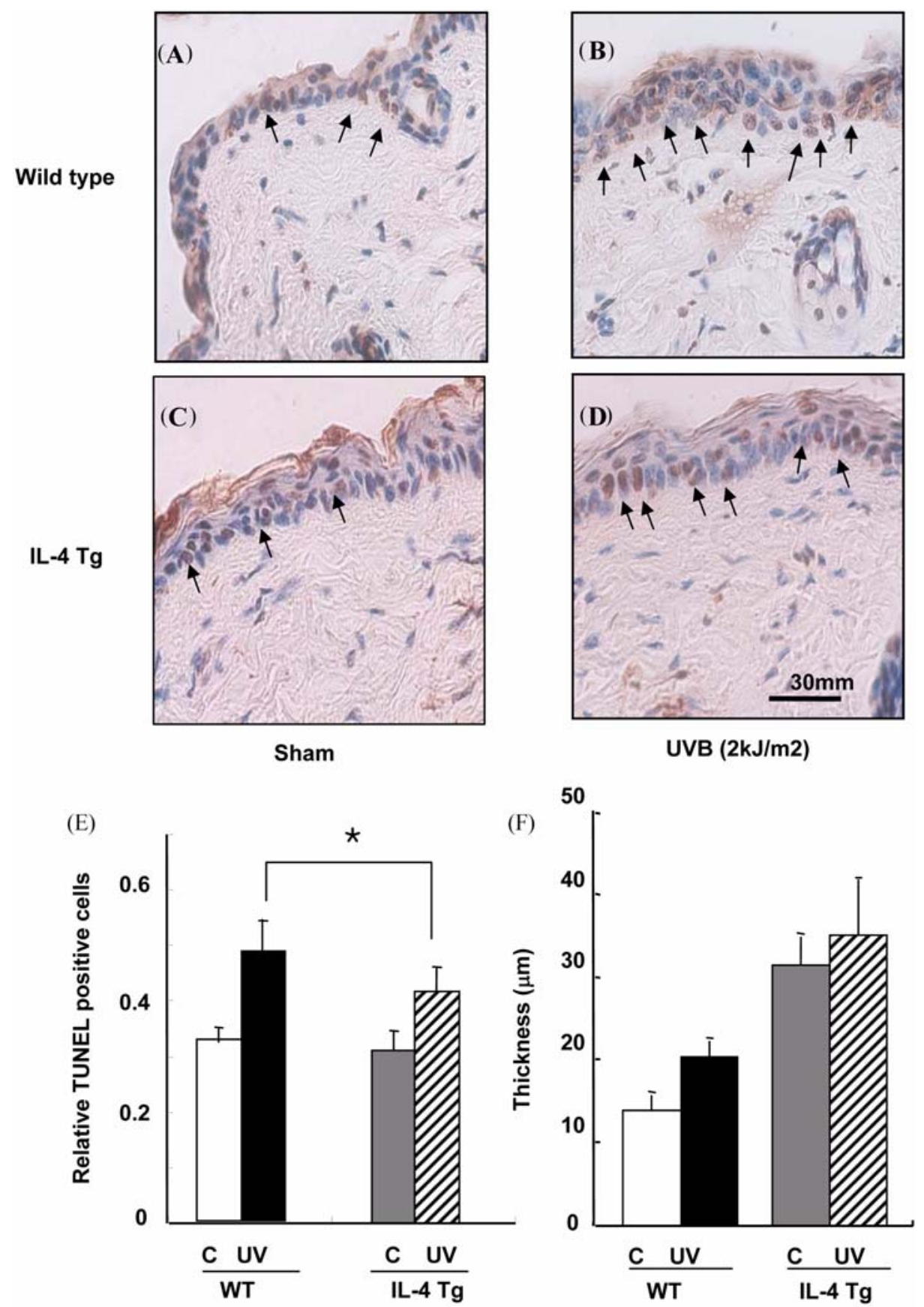

Fig. 5. Detection of apoptosis after UVB irradiation. Skin biopsies were taken $24 \mathrm{~h}$ after irradiation with UVB $\left(2 \mathrm{~kJ} / \mathrm{m}^{2}\right)$ and were used for TUNEL staining (A-D). (E) The number of TUNEL positive cell was counted in equal area of six randomly selected regions. Relative TUNEL positive cells means the fraction of TUNEL positive cells per total cells counted in each field. (F) Ear thickness $24 \mathrm{~h}$ after UVB irradiation. The value for each group is the mean $+/-\mathrm{SD}\left({ }^{*} p<0.1\right)$.

As shown in Fig. 7A, TUNEL assay revealed decrease of apoptotic cells in Pam212/IL-4 clones, supporting the previous in vivo data. Furthermore, FACS analysis to detect annexin Vpositive cells also showed significantly reduced apoptosis (27.1\% vs. $1.33 \%)$ in Pam 212/IL-4 clones following UVB irradiation. Taken together, it is suggested that IL-4 has the protective effect on keratinocytes from UVB-induced apoptosis.

\section{Discussion}

In this study, we investigated the role of IL-4 in skin using transgenic mice, which is overexpressing IL-4 in keratinocytes. IL-4 has diverse functions, which affect cell growth, survival, differentiation, and regulation of immune response (Keegan et al., 1998; Hart et al., 1999; Boothby et al., 2001; Lutz, 2004). 
(A)

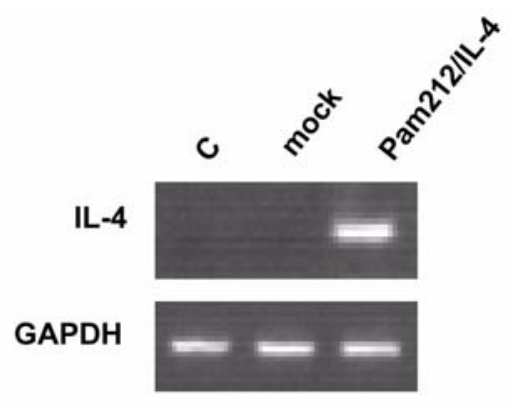

(B)

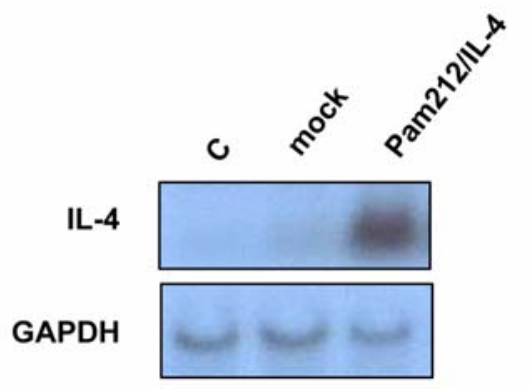

(C)

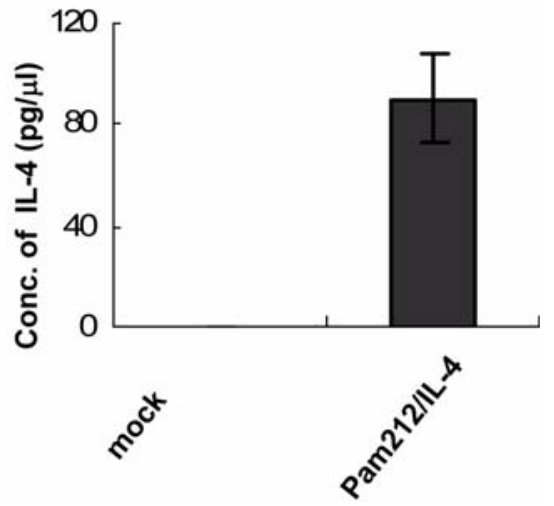

Fig. 6. IL-4 overexpressing stable cell clones (Pam212/IL-4). Mouse keratinocytes, Pam212, were transfected as described in Materials and Methods. The expression of IL-4 in the selected cell clones was confirmed by RT-PCR (A), northern blot analysis (B), and ELISA (C).

In skin, IL-4 has been considered as an important factor in pathogenesis of atopic dermatitis (AD) as IL-4 was shown to be expressed in lesional skin derived from AD patients (Ricci, 1994; Shirakawa et al., 2000; Izuhara et al., 2000). Chan et al. (2001) demonstrated that transgenic mice, which overexpress IL-4 in epidermis, spontaneously developed a skin disease showing symptoms of human AD including xerosis, conjunctivitis, inflammatory skin lesions, and infiltration of immune cells in epidermis. On the other hand, Elbe-Burger et al. (2002) showed that the consequence of IL-4 overexpression in skin had no signs of spontaneous skin disease, but displayed histopathological changes involving both the epidermis and the dermis, including epidermal hyperplasia, hyperkeratosis, an increase of Langerhans cells, upregulation of specific
(A)

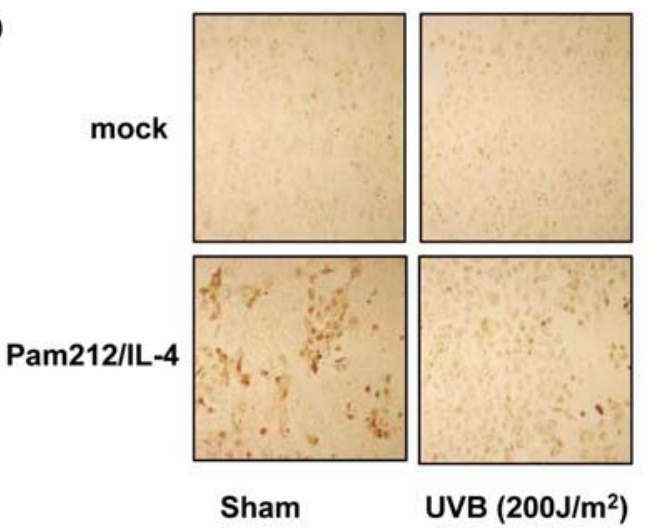

(B)

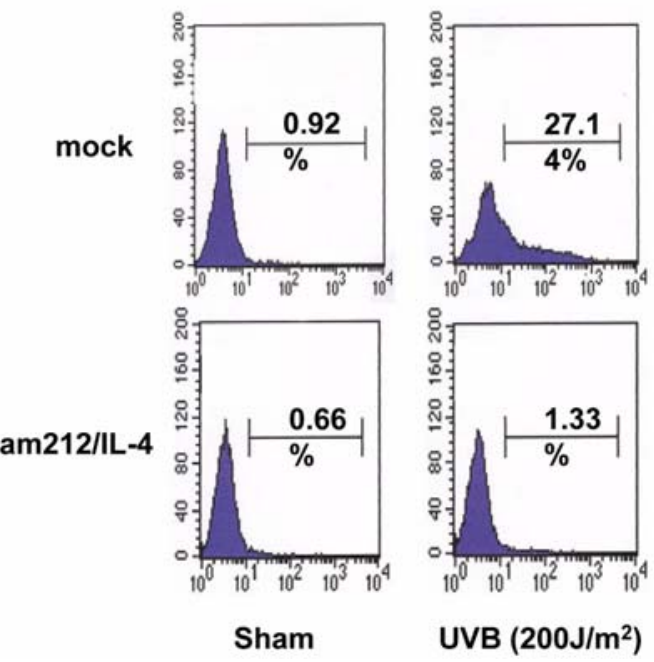

Fig. 7. Reduction of apoptosis in Pam $212 / \mathrm{IL}-4$. (A) TUNEL assay. Cells were UVB irradiated at $200 \mathrm{~mJ} / \mathrm{m}^{2}$ and TUNEL assay was conducted $24 \mathrm{~h}$ after irradiation. (B) Detection of annexin-V positive cells. UVB-irradiated cells were harvested and incubated with FITC-conjugated annexin-V antibody for $1 \mathrm{~h}$ and flow cytometry was conducted.

surface markers on a subpopulation of Langerhans cells, proliferation of fibroblasts, an accumulation of collagen and mast cells in the dermis, and a reduction of adipocytes. In our study, no gross skin abnormality was observed in IL-4 Tg mice. However, histological analysis of IL-4 Tg mice revealed thickened epidermis as well as acanthosis, hyperkeratosis, parakeratosis. In addition, the level of MHC class II was increased in epidermis of IL-4 Tg mice compared with wildtype. Since it was previously reported that $\mathrm{IL}-4$ overexpression in B cells led to increased expression of MHC class II, resulting in the enhancement of antigen presenting capacity (Poncet et al., 1999; Stuart et al., 2000), we anticipate IL-4 overexpression in keratinocytes in mouse skin led to increased expression of MHC class II in epidermal cells.

It was previously suggested by Elbe-Burger et al. (2002) that, in transgenic mice in which IL-4 is ubiquitously expressed, the number of Langerhans cells (LC) was increased due to the impaired efflux from the epidermis to the dermis or inhibition 
of migratory activity. In our study on the migratory activity of LC though induction of contact hypersensitivity (CHS), however, IL-4 Tg mice did not show any differential response compared with that of wild-type control (data not shown), implying that the migratory activity of LC in skin of IL-4 Tg mice was not impaired. It is yet unknown whether the skin-specific expression of IL-4 is responsible for the different CHS response compared to that of systemic expression as reported by Elbe-Burger et al. (2002).

It is well recognized that UVB (290-320nm) irradiation can induce several types of epidermal injury, including sunburn cell formation. These injuries are considered to be associated with skin carcinogenesis, skin aging and immune-suppression (Berg et al., 1996; Schwartz et al., 1998; Cooper et al., 2003; Schwartz et al., 2005a). UVB-induced immunosuppression, in fact, has been used for clinical applications. IL-4 was reported to mediate UVB-induced immunosuppression using $\mathrm{IL}-4^{-/}$ mouse system, in which delayed hypersensitivity $(\mathrm{DH})$ was suppressed (el-Ghorr and Norval, 1997). In addition, apoptosis of antigen-presenting cells by UVB-induced suppressor T cells was suggested to be involved in UVB-induced immunosuppression (Schwarz et al., 1998b). One possible explanation for IL-4 in immunosuppression might be the secretion of immune suppressive cytokines IL-4 and IL-10 by UVB-induced apoptotic T cells (Aubin and Mousson, 2004).

Meanwhile, the study presented here demonstrated that ectopic expression of IL-4 in keratinocytes of mouse skin and in keratinocyte cell line was able to suppress UVB-induced apoptois. There are several reports showing that IL-4 acts as a survival factor in a number of different biological environments (Mori et al., 2000; Morris et al., 2002). Zamorano et al. (2001) suggested that IL-4-induced protection from apoptosis might be mediated through cooperation with the NF- $\kappa$ B family of transcription factors. Also, IL-4 protects endothelial cells from apoptosis though activation of PI3K/AKT pathway (Grehan et al., 2005). Meanwhile, various factors such as ROS, FasL and p53 are involved in UVB-induced apoptosis and intense studies to identify factor(s) to regulate UVBinduced response have been conducted (S Berg et al., 1996; Bang et al., 2003; Dazard et al., 2003; Murahashi et al., 2003; Schimizu et al., 2005). Although it is not established yet whether both immunosuppressive and anti-apoptotic roles of IL-4 are directly linked to each other, cell-type specificity is also likely to be one responsible factor for either function.

Keratinocytes protect body from various environmental factors and is the primary target for UVB-induced responses. Keratinocytes produce various kinds of cytokines, which affect other cells and organs, mediating cellular growth and differentiation as well as inflammation and immune reactions (McKenzie et al., 1990; Akiba et al., 2002; Dazard et al., 2003; Takasawa et al., 2005). The results presented here provide evidences that IL-4 in keratinocytes plays an antiapoptotic role against UVB irradiation. Currently, investigation is on-going to elucidate the exact mechanism of the protective effect of IL-4 in keratinocytes against UVB-induced apoptosis.
In conclusion, we generated transgenic mice expressing IL4 in skin showing no gross clinical abnormalities. However, in IL-4 Tg mice, increased infiltration of immune cells in skin was detected in addition to histological abnormalities such as acanthosis, hyperkeratosis, and parakeratosis. Furthermore, IL-4 protected keratinocytes in skin from apoptosis induced by UVB irradiation and this was confirmed in vitro using stable cell clones. These results provide an insight that IL-4 may be an important regulator in cutaneous immune system against UVB.

Acknowledgments This work was supported by a Next Generation Growth Engine Program Grant from the Korean Science and Engineering Foundation and Korean Ministry of Science and Technology.

\section{References}

Akiba, H., Kehen, J., Ducluzeau, M. T., Krasteva, M., Horand, F., Kaiserlian, D., Kaneko, F. and Nicolas, J. F. (2002) Skin inflammation during contact hypersensitivity is mediated by early recruitment of $\mathrm{CD} 8+\mathrm{T}$ cytotoxic 1 cells inducing keratinocyte apoptosis. J. Immunol. 15, 3079-3087.

Aubin, F. and Mousson, C. (2004) Ultraviolet light-induced regulatory (suppressor) $\mathrm{T}$ cells: an approach for promoting induction of operational tolerance? Transplantation 77, 29-31.

Bang, B., Gniadecki, R., Larsen, J. K., Baadsgaard, O. and Skov, L. (2003) In vivo UVB irradiation induces clustering of Fas (CD95) on human epidermal cells. Exp. Dermatol. 12, 791798.

Berg, R. J., van Kranen, H. J., Rebel, H. G., de Vries, A., van Vloten, W. A., Van Kreijl, C. F., van der Leun, J. C. and de Gruijl, F. R. (1996) Early p53 alterations in mouse skin carcinogenesis by UVB radiation: immunohistochemical detection of mutant $\mathrm{p} 53$ protein in clusters of preneoplastic epidermal cells. Proc. Natl. Acad. Sci. USA 93, 274-278.

Boothby, M., Mora, A. L., Aronica, M. A., Youn, J., Sheller, J. R., Goenka, S. and Stephenson, L. (2001) IL-4 signaling, gene transcription regulation, and the control of effector $\mathrm{T}$ cells. Immunol. Rev. 23, 179-191.

Chan, L. S., Robinson, N. and Xu, L. (2001) Expression of interleukin-4 in the epidermis of transgenic mice results in a pruritic inflammatory skin disease: an experimental animal model to study atopic dermatitis. J. Invest. Dermatol. 117, 977983.

Cleaver, J. E. and Crowley, E. (2002) UV damage, DNA repair and skin carcinogenesis. Front. Biosci. 1, d1024-1043.

Cooper, K. D., Baron, E. D. and Matsui, M. S. (2003) Implications of UV-induced inflammation and immunomodulation. Cutis 72, 11-15.

Dazard, J. E., Gal, H., Amariglio, N., Rechavi, G., Domany, E. and Givol, D. (2003) Genome-wide comparison of human keratinocyte and squamous cell carcinoma responses to UVB irradiation: implications for skin and epithelial cancer. Oncogene 15, 2993-3006.

Elbe-Burger, A., Egyed, A., Olt, S., Klubal, R., Mann, U., Rappersberger, K., Rot, A. and Stingl, G. (2002) 
Overexpression of IL-4 alters the homeostasis in the skin. $J$. Invest. Dermatol. 118, 767-778.

El-Ghorr, A. A. and Norval, M. (1997) The role of interleukin-4 in ultraviolet B light-induced immunosuppression. Immunology 92, 26-32.

Grehan, J. F., Levay-Young, B. K., Fogelson, J. L., FrancoisBongarcon, V., Benson, B. A. and Dalmasso, A. P. (2005) IL-4 and IL-13 induce protection of porcine endothelial cells from killing by human complement and from apoptosis though activation of a phosphatidylinositide 3-kinase/Akt pathway. $J$. Immunol. 175, 1903-1910.

Hart, P. H., Bonder, C. S., Balogh, J., Dickensheets, H. L., Donnelly, R. P. and Finlay-Jones, J. J. (1999) Differential responses of human monocytes and macrophages to IL-4 and IL-13. J. Leukoc. Biol. 66, 575-578.

Izuhara, K., Yanagihara, Y., Hamasaki, N., Shirakawa, T. and Hopkin, J. M. (2000) Atopy and the human IL-4 receptor alpha chain. J. Allergy. Clin. Immunol. 106, 65-71.

Keegan, A. D. and Zamorano, J. (1998) Regulation of gene expression, growth, and cell survival by IL-4: contribution of multiple signaling pathways. Cell. Res. 8, 1-13.

Kulms, D. and Schwarz, T. (2002) Mechanisms of UV-induced signal transduction. J. Dermatol. 29, 189-196.

Liu, Z., Liu, Q., Pesce, J., Anthony, R. M. , Lamb, E., Whitmire, J., Hamed, H., Morimoto, M., Urban, J. F. and Jr. Gause, W. C. (2004) Requirements for the development of IL-4-producing $\mathrm{T}$ cells during intestinal nematode infections: what it takes to make a Th2 cell in vivo. Immunol. Rev. 201, 57-74.

Lorentz, A. and Bischoff, S. C. (2001) Regulation of human intestinal mast cells by stem cell factor and IL-4. Immunol. Rev. 179, 57-60.

Lorentz, A., Wilke, M., Sellge, G., Worthmann, H., Klempnauer, J., Manns, M. P. and Bischoff, S. C. (2005) IL-4-induced priming of human intestinal mast cells for enhanced survival and Th2 cytokine generation is reversible and associated with increased activity of ERK $1 / 2$ and c-Fos. J. Immunol. 174, 6751-6756.

Lutz, M. B. (2004) IL-3 in dendritic cell development and function: a comparison with GM-CSF and IL-4. Immunobiology 209, 79-87.

McKenzie, R. C. and Sauder, D. N. (1990) The role of keratinocyte cytokines in inflammation and immunity. J. Invest. Dermatol. 95, 105-107.

Mori, M., Morris, S. C., Orekhova, T., Marinaro, M., Giannini, E. and Finkelman, F. D. (2000) IL-4 promotes the migration of circulating B cells to the spleen and increases splenic B cell survival. J. Immunol. 164, 5704-5712.

Morris, S. C., Dragula, N. L. and Finkelman, F. D. (2002) IL-4 promotes Stat6-dependent survival of autoreactive B cells in vivo without inducing autoantibody production. J. Immunol.
169, 1696-1704.

Murahashi, H., Azuma, H., Zamzami, N., Furuya, K. J., Ikebuchi, K., Yamaguchi, M., Yamada, Y., Sato, N., Fujihara, M., Kroemer, G. and Ikeda, H. (2003) Possible contribution of apoptosis-inducing factor (AIF) and reactive oxygen species (ROS) to UVB-induced caspase-independent cell death in the T cell line Jurkat. J. Leukoc. Biol. 73, 399-406.

Poncet, P., Arock, M. and David, B. (1999) MHC class IIdependent activation of $\mathrm{CD} 4+\mathrm{T}$ cell hybridomas by human mast cells though superantigen presentation. J. Leukoc. Biol. 66, $105-112$.

Ricci, M. (1994) IL-4: a key cytokine in atopy. Clin. Exp. Allergy 24, 801-812.

Schwarz, A., Maeda, A., Kernebeck, K., van Steeg, H., Beissert, S. and Schwarz, T. (2005a) Prevention of UV radiation-induced immunosuppression by IL-12 is dependent on DNA repair. $J$. Exp. Med. 201, 173-179.

Schwarz, A., Grabbe, S., Gross-Heitmeyer, K., Roters, B., Riemann, H., Luger, T.A., Trinchieri, G., and Schwaz, T. (1998b) Ultraviolet light-induced immune tolerance is mediated via the Fas/FasL system. J. Immunol. 160, 4262-4270.

Schwarz, A. and Schwarz, T. (2002c) Molecular determinants of UV-induced immunosuppression. Exp. Dermatol. 11, 9-12.

Schwarz, T. (1998) Mechanisms of UV-induced immunosuppression. Link between UV-induced tolerance and apoptosis. Eur. $J$. Dermatol. 8, 196-197.

Shirakawa, I., Deichmann, K. A., Izuhara, I., Mao, I., Adra, C. N. and Hopkin, J. M. (2000) Atopy and asthma: genetic variants of IL-4 and IL-13 signaling. Immunol. Today 21, 60-64.

Shimizu, H., Banno, Y., Sumi, N., Naganawa, T., Kitajima, Y. and Nozawa, Y. (1999) Activation of p38 mitogen-activated protein kinase and caspases in UVB-induced apoptosis of human keratinocyte HaCaT cells. J. Invest. Dermatol. 112, 769-774.

Stack, R. M., Thompson, C. B. and Fitch, F. W. (1998) IL-4 enhances long-term survival of CD28-deficient $\mathrm{T}$ cells. $J$. Immunol. 160, 2255-2262.

Stuart, P. M., Zlotnik, A. and Woodward, J. G. (2000) Atopy and the human IL-4 receptor alpha chain. J. Allergy. Clin. Immunol. 106, 65-71.

Takasawa, R., Nakamura, H., Mori, T. and Tanuma, S. (2005) Differential apoptotic pathways in human keratinocyte HaCaT cells exposed to UVB and UVC. Apoptosis 10, 1121-1130.

Ullrich, S. E. and Schmitt, D. A. (2000) The role of cytokines in UV-induced systemic immune suppression. J. Dermatol. Sci. 23, 10-12.

Zamorano, J., Mora, A. L., Boothby, M. and Keegan, A. D. (2001) NF-kappa B activation plays an important role in the IL-4-induced protection from apoptosis. Int. Immunol. 13, 1479-1487. 